\title{
Common Zeros of Two Bessel Functions
}

\author{
By T. C. Benton and H. D. Knoble
}

\begin{abstract}
There is a theorem that two Bessel functions $J_{\mu}(x)$ and $J_{\nu}(x)$ can have no common positive zeros if $\mu$ is an integer and $\nu=\mu+m$ where $m$ is an integer, but this does not preclude the possibility that for unrestricted real positive $\mu$ and $\nu$ not differing by an integer, the two functions $J_{\mu}(x)$ and $J_{\nu}(x)$ can have common zeros. An example is found where two such functions have two positive zeros in common.
\end{abstract}

1. Introduction. The Bessel functions $J_{\nu}(x)$, where $v$ is a real number, is known to have an infinity of positive zeros $j_{\nu, 1}, j_{\nu, 2}, j_{\nu, 3}, \ldots, j_{\nu, s}, \ldots$ If $\nu$ is variable, $j_{\nu, s}$ is a continuous increasing function of $\nu$. As $n \rightarrow \infty,\left(j_{\nu, n+1}-j_{\nu, n}\right) \rightarrow \pi$. There is a theorem that $J_{\nu}(x)$ and $J_{\nu+n}(x), n$ an integer, have no common zeros. Proofs of these facts are in [6].

The theorem just cited does not preclude the possibility that if $\mu-\nu$ is not an integer, the functions $J_{\nu}(x)$ and $J_{\mu}(x)$ may have zeros in common. It is quite obvious that two functions can have one positive zero in common because $\nu$ can be determined so that $j_{\nu, 1}$ coincides with a particular $j_{\mu, s}$ where $\mu<\nu$. An inspection of tables [3] shows that for some $\nu$ between 3.5 and $4, J_{\nu}(x)$ will have its first zero $j_{\nu, 1}=j_{1,2}$.

It is the purpose of the present investigation to show that there exist values $\mu$ and $\nu$ such that $J_{\mu}(x)$ and $J_{\nu}(x)$ have at least two positive zeros in common.

2. Estimations for an Example. By searching tables [3], [5] it was found that the interval between $j_{\mathbf{0 , 1 2}}=36.9170984$ and $j_{\mathbf{0}, 15}=46.3411884$ was nearly equal to the interval from $j_{\mathbf{3 0 , 1}}$ to $j_{\mathbf{3 0 , 3}}$ which is approximately 9.354. This is less than $j_{\mathbf{0 , 1 5}}-$ $j_{\mathbf{0 , 1 2}}=9.42409$. But the interval from $j_{\mathbf{3 1 , 1}}$ to $j_{\mathbf{3 1 , 3}}$ has $j_{\mathbf{3 1 , 3}}-j_{\mathbf{3 1 , 1}}=9.4249$ which is greater than $j_{0,15}-j_{0,12}$.

This suggests that if $\mu$ is determined so that $j_{\mu, 1}=36.917$, then $j_{\mu, 3}$ should be close to $j_{0,15}$. It happens that $j_{\mu, 3} \cong 46.3258<j_{0,15}=46.3412$. Now if $\mu$ is slightly increased $j_{\mu, 3}-j_{\mu, 1}$ will be increased. This can be shown by using the series found by F. W. J. Olver [4]:

$$
\begin{aligned}
j_{\nu, 1} & =\nu+1.8557571 \nu^{1 / 3}+1.0331503 \nu^{-1 / 3}-0.003974 \nu^{-1}-\cdots, \\
j_{\nu, 3} & =\nu+4.3816712 \nu^{1 / 3}+5.7597129 \nu^{-1 / 3}-0.226068 \nu^{-1}-\cdots, \\
j_{\nu, 3}-j_{\nu, 1} & =2.5259141 \nu^{1 / 3}+4.7265626 \nu^{-1 / 3}-0.222094 \nu^{-1}, \\
\frac{d}{d \nu}\left(j_{\nu, 3}-j_{\nu, 1}\right) & =0.8419714 \nu^{-2 / 3}-1.5455209 \nu^{-4 / 3}+0.222094 \nu^{-2} \\
& =0.8419714 \nu^{-2}\left\{\nu^{4 / 3}-1.8712285 \nu^{2 / 3}+0.2637786\right\} .
\end{aligned}
$$


This will be $>0$ if $\nu^{2 / 3}>1.7176599$ or $\nu>2.2511569$. Hence, $j_{\nu, 3}-j_{\nu, 1}$ will be increasing if $\nu>2.26$. Now if 0 is increased to a small value $\nu$ where $0<\nu<1, j_{0,15}$ $-j_{0,12}$ will also be increased but since this difference is much closer to the limit $3 \pi$ its rate of increase will be much less than the rate of increase of $j_{\mu, 3}-j_{\mu, 1}$. However, for $J_{31}(x), J_{31,1}=37.1581130$ and $j_{31,3}=46.58280123$; and if $v$ is found so that $j_{\nu, 12}=j_{31,1}$, the value is $\nu=0.1536508$. Then $j_{\nu, 15}=46.5822752$. So here $j_{31,3}$ $-j_{31,1}>j_{\nu, 15}-j_{\nu, 12}$.

Since the zeros are continuous functions of the order, the difference of two zeros is also, and it has been shown that $j_{\mu, 3}-j_{\mu, 1}$ changes from less than $j_{\nu, 15}-$ $j_{\nu, 12}$ to greater than $j_{\nu, 15}-j_{\nu, 12}$ where the value of $\nu$ has been chosen to make $j_{\mu, 1}=j_{\nu, 12}$. Then on account of continuity there must be a value of $\nu$ for which $j_{\mu, 3}-j_{\mu, 1}=j_{\nu, 15}-j_{\nu, 12}$ and since $j_{\mu, 1}=j_{\nu, 12}$ we must have $j_{\mu, 3}=j_{\nu, 15}$.

Thus, for some $\mu$ between 30 and 31 and some $\nu$ between 0 and $1, J_{\mu}(x)$ and $J_{\nu}(x)$ will have two positive zeros in common.

3. Computations. During the course of preparing this paper, it was useful to be able to calculate the roots of the Bessel functions accurate at least to ten significant digits. Capitalizing on the works of Gautschi [2] to evaluate the Bessel functions accurately and Brent [1] to compute a zero of an arbitrary function, an IBM 370 FORTRAN computer program was written to compute distinct values of $\mu$ and $\nu$ such that $J_{\mu}(x)=0$ and $J_{\nu}(x)=0$. The program is an implementation of the following algorithm:

Step 1. Input the eight quantities, $\mu_{a}, \mu_{b}, v_{a}, v_{b}, x_{a}, x_{b}, z_{a}, z_{b}$; more specifically:

1.1 define endpoints for the interval $\nu \in\left[v_{a}, \nu_{b}\right]$ for the Bessel functions of order $\nu$.

1.2 define endpoints for the interval $z \in\left[z_{a}, z_{b}\right] \ni J_{\nu}(z)=0$.

1.3 define endpoints for the interval $\mu \in\left[\mu_{a}, \mu_{b}\right] \ni J_{\mu}(z)=0$ and $\mu \notin$ $\left[v_{a}, v_{b}\right]$.

1.4 define endpoints for the interval $x_{1}, x_{2} \in\left[x_{a}, x_{b}\right] \ni J_{\nu}\left(x_{1}\right)=0, J_{\mu}\left(x_{2}\right)$ $=0, z \notin\left[x_{a}, x_{b}\right]$.

Step 2. Define four versions of Brent's algorithm say $B_{1}, B_{2}, B_{3}, B_{4}$ such that given a specific value of $\nu$ and the respective intervals from Step 1 for $z, x_{1}, x_{2}, \mu$ :

2.1 $B_{1}$ solve for $z$ in the equation $J_{\nu}(z)=0$.

$2.2 B_{2}$ solve for $x_{1}$ in the equation $J_{\nu}\left(x_{1}\right)=0$.

$2.3 B_{3}$ solve for $\mu$ in the equation $J_{\mu}(z)=0$ given $z$ from 2.1 .

$2.4 B_{4}$ solve for $x_{2}$ in the equation $J_{\mu}\left(x_{2}\right)=0$ given $\mu$ from 2.3. If any version fails to converge, terminate with appropriate message.

Step 3. Define the function $F(v)=\left|x_{1}-x_{2}\right|$ where $x_{1}$ and $x_{2}$ are derived by performing Steps 2.1 through 2.4.

Step 4. Given the interval for $\nu$, use a version of Brent's algorithm, say $B_{F}$, to solve for $\nu$ in the equation $F(\nu)=0$. If $B_{F}$ fails to converge, terminate with appropriate message.

Step 5. Output the final value of $\mu, \nu, z$ and $x=x_{1}=x_{2}$, then terminate. 
For example: Given the input values $\mu_{a}=30.9, \mu_{b}=31 ; v_{a}=0, v_{b}=1 ; z_{a}=$ $37, z_{b}=38 ; x_{a}=46, x_{b}=47$; the program output the values

$$
\begin{aligned}
& \mu=30.99239611, \quad \nu=0.1485098818, \\
& z=j_{\mu, 1}=j_{\nu, 12}=37.15005920 \\
& x=j_{\mu, 3}=j_{\nu, 15}=46.57421695 .
\end{aligned}
$$

These figures show that the two Bessel functions $J_{\mu}(x)$ and $J_{\nu}(x)$ have a pair of positive zeros which are common to the two functions.

On account of the continuity there must be such a coincidence and this calculation gives the orders of the two functions and the position of the common zeros to eight places of decimals (the computations were carried out to 13 places).

4. Conclusion. Having constructed a computer program to perform these operations, the possible pairs of Bessel functions which have at least two zeros in common can be studied.

This raises a number of interesting questions. Perhaps the most interesting one is whether two functions can have more than two zeros in common. If two functions do have more than two zeros in common, they will have several pairs of common zeros; and if this occurs, the same pair of values of $\mu$ and $\nu$ will appear for different pairs of values of the zeros. Therefore, it seems that an answer to this question may come from a systematic study of pairs.

This idea has led to finding numerous examples by applying the computer program-about 30 examples are now known, but none have so far given more than one pair of common zeros.

The authors are grateful for a grant from the Mathematics Department at The Pennsylvania State University and for other time on the computer at the University Computer Center.

226 South Barnard Street

State College, Pennsylvania 16801

The Computation Center

Pennsylvania State University

University Park, Pennsylvania 16802

1. R. P. BRENT, “An algorithm with guaranteed convergence for finding a zero of a function," Comput. J., v. 14, 1971, pp. 422-425; in particular see the FORTRAN implementation en titled "ZBRENT" by International Mathematical and Statistical Libraries, Inc., 5th ed., Houston, 1975.

2. W. GAUTSCHI, "Algorithm 236, Bessel functions of the first kind," Comm. ACM, v. 7, 1964, pp. 479-480.

3. HARVARD COMPUTATION LABORATORY, Tables of Bessel Functions, 1947-1951 Annals, vols. III-XIV, Harvard Univ. Press, Cambridge, Mass.

4. F. W. J. OLVER, “... Evaluation of zeros of Bessel functions...," Proc. Cambridge Philos. Soc., v. 47, 1951, pp. 699-712.

5. ROYAL SOCIETY, Mathematical Tables 7, Bessel Functions III, edited by F. W. J. Olver, published for the Royal Society by Cambridge Univ. Press, 1960.

6. G. N. WATSON, Bessel Functions, 4th ed., Cambridge Univ. Press, 1944. 